\title{
The associations between bone mineral density and cerebral white matter hyperintensity in elderly stroke patients
}

\author{
Ji-Sun Kim ${ }^{1}$, Minjik Kim ${ }^{1}$, Sung Hoon Kang ${ }^{1}$, Kyungmi Oh ${ }^{1}$, Sangil Suh ${ }^{2}$, Woo-Keun Seo ${ }^{3,4}$ \\ ${ }^{1}$ Department of Neurology, Korea University Guro Hospital, Korea University College of Medicine, Seoul, Korea \\ ${ }^{2}$ Department of Radiology, Korea University Guro Hospital, Korea University College of Medicine, Seoul, Korea \\ ${ }^{3}$ Department of Neurology, Samsung Medical Center, Sungkyunkwan University School of Medicine, Seoul, Korea \\ ${ }^{4}$ Department of Digital Health, Samsung Advanced Institute for Health Sciences \& Technology (SAIHST), Sungkyunkwan University, \\ Seoul, Korea
}

Received: July 20, 2018

Revised: July 29, 2018

Accepted: August 1, 2018

Corresponding author:

Woo-Keun Seo

Department of Neurology,

Samsung Medical Center,

Sungkyunkwan University

School of Medicine, 81 Irwon-ro,

Gangnam-gu, Seoul 06351, Korea

Tel: +82-2-3410-0799

E-mail: mcastenosis@gmail.com

\section{ABSTRACT}

Purpose: Osteoporosis is an important senile disease and has significant relationship with ischemic stroke and cerebral small vessel disease (SVD). Correlation between bone mineral density (BMD) and cerebral white matter hyperintensity on magnetic resonance imaging were analyzed to investigate the relationship between osteoporosis and cerebral SVD.

Methods: Medical records of acute stroke patients with age $\geq 65$ years were retrospectively collected from single center. Cerebral white matter hyperintensity was classified into two categories, periventricular white matter hyperintensity (PWMH) and deep white matter hyperintensity (DWMH), and the severity was graded according to maximal lesion size. Association between the clinical factors including BMD and the severity of cerebral white matter hyperintensity was analyzed.

Results: Four hundred eight patients were included in the study. High severity grade of both MWMH and DWMH was independently correlated with low BMD. Additionally, high PWMH grade was correlated with old age and high serum homocysteine. High DWMH grade was correlated with old age, atrial fibrillation history and high serum total calcium. Conclusion: Low BMD was associated with a high severity of cerebral white matter hyperintensity in elderly Asian stroke patients, independent of other clinical factors.

Keywords: Bone density; Cerebral white matter hyperintensity; Small vessel disease

\section{INTRODUCTION}

This is an Open Access article distributed under the terms of the Creative Commons Attribution Non-Commercial License (http:// creativecommons.org/licenses/ by-nc/4.0/).
Osteoporosis is a huge burden of the elderly population that annually causes millions of fractures worldwide [1]. In addition, osteoporosis has close relationship with systemic vascular diseases such as ischemic stroke. Ischemic stroke and osteoporosis have mutual relationship in that osteoporosis could deteriorate by stroke and stroke patients with osteoporosis showed 
poor clinical outcome [2-4]. A resent epidemiologic study revealed that osteoporosis was associated with the risk of ischemic stroke and accompanied meta-analysis also verified the association between osteoporosis and the risk of ischemic stroke [5].

Osteoporosis also had association with cerebral small vessel disease (SVD), a important cause of ischemic stroke. A recent population-based study showed osteoporosis have an association with cerebral SVD [6]. Other study demonstrated the association between osteoporosis and cerebral white matter changes conducted based on computed tomography (CT) findings [7]. Although the rating scale of cerebral white matter changes applicable to both $\mathrm{CT}$ and magnetic resonance imaging (MRI) was available, MRI is still superior in sensitivity especially for detecting small sized changes [8]. Thus, more detailed validations using MRI is requested.

Cerebral white matter hyperintensity is one of the important MRI findings of cerebral SVD [9]. This study aimed to investigate the association between osteoporosis and cerebral SVD, by analyzing correlation of bone mineral density (BMD) and severity of cerebral white matter hyperintensity on MRI.

\section{METHODS}

\section{Subjects}

Data were extracted from a prospectively collected hospital-based stroke registry (Korea University Stroke Registry-Guro arm; KUSR-G) from January 2008 to September 2014. Details of
KUSR were presented in elsewhere [10]. In brief, KUSR have collected the data about acute stroke patients within seven days from the onset of the stroke such as demographic data, vascular risk factors, laboratory findings, and imaging features. Diagnosis of stroke is based on focal neurological symptoms with relevant brain imaging findings. Among the patient registered in KUSR during study period, we selected the patients who met the following criteria; age $\geq 65$ years, measurement of BMD, and available fluid attenuated inversion recovery (FLAIR) images on MRI. We sequentially excluded patients who had been prescribed for anti-osteoporotic medications such as alendronate, cholecalciferol, calcitriol, calciumcitrate, or calciumcarbonate. The study protocol was approved and supervised by the Institutional Review Boards of Korea University Medical Center (KUGH-15111). Informed consent was waived by the committee because of the retrospective study design.

\section{Bone densitometry and magnetic resonance images}

BMD was measured by dual-energy $\mathrm{X}$-ray absorptiometry (Hologic Discovery A, Belford, MA, USA) for lumbar spine and presented as T-score: T-score = (individual BMD-reference BMD)/ reference SD. MRI examination was performed using 1.5 Tesla magnetic resonance (MR) equipment (Sonata, Siemens, Erlangen, Germany) or 3.0 Tesla MR equipment (TrioTim, Siemens). All patients underwent FLAIR imaging (TR 10,000 ms, TE 95 ms, slice thickness $5 \mathrm{~mm}$, FOV $384 \times 218$, no gap for 3.0 Tesla MR; or TR 8,000 ms, TE 108 ms, slice thickness 5 mm, FOV 256 $\times 192$, no gap for 1.5 Tesla MR).
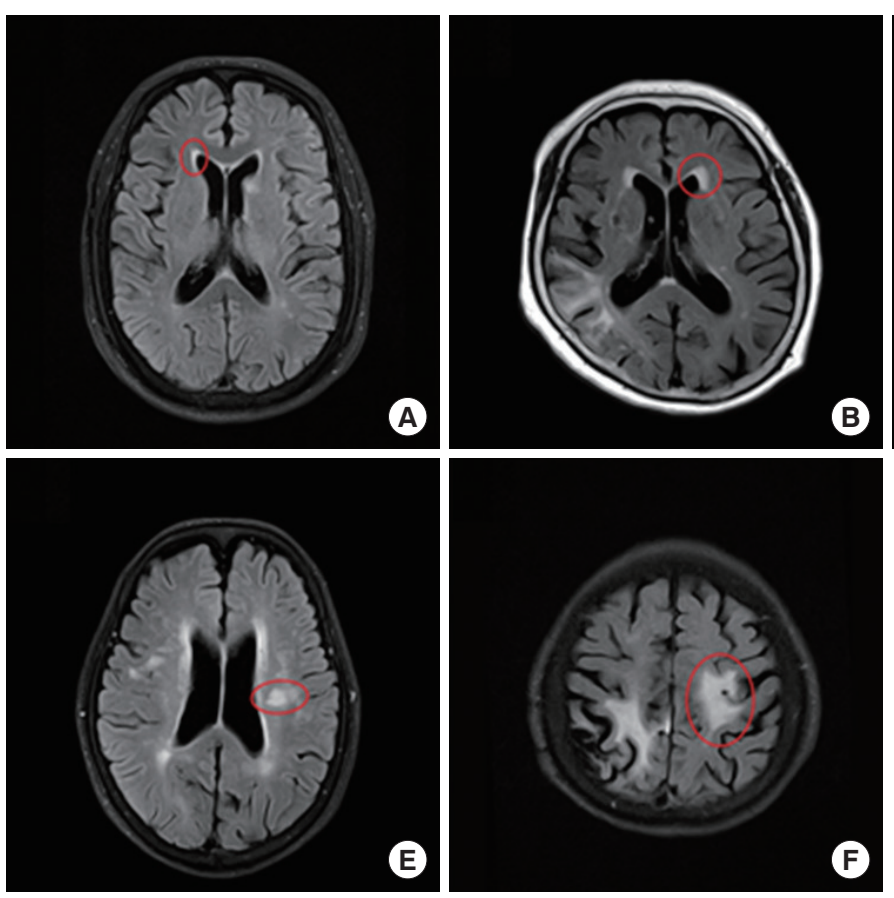
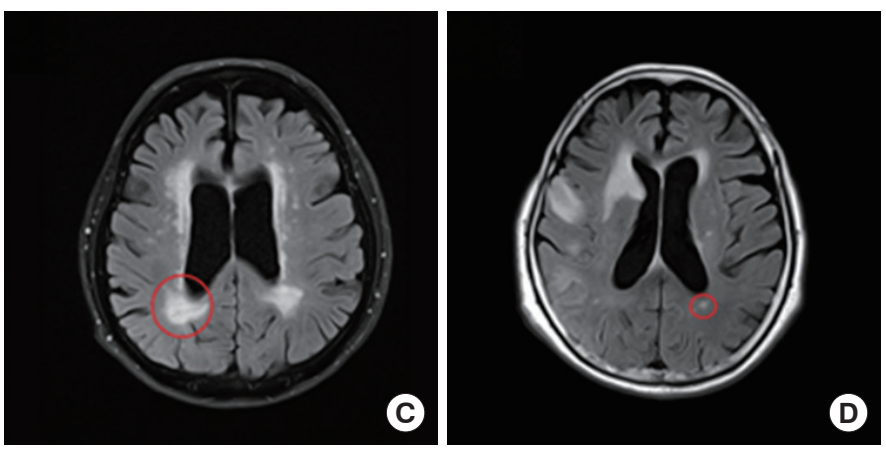

Fig. 1. Measuring method and grading of cerebral white matter hyperintensity in fluid attenuated inversion recovery (FLAIR) images.

$(\mathrm{A}-\mathrm{C})$ These were the examples of periventricular white matter hyperintensity $(\mathrm{PWMH})$, grade 1,2 , and 3 , respectively. Among the periventricular caps, longest one is selected and used for the grading. (D-F) These were the examples of deep white matter hyperintensity (DWMH), grade 1, 2, and 3 serially. When measuring PWMH and DWMH, acute infarcted areas were excluded. In case of (D), the subject had acute infarction in right temporo-occipital lobes; hence, FLAIR high signal lesions within the infarcted area were not used for the measurement. 


\section{Measurement of white matter hyperintensity on MRI}

White matter hyperintensity was assessed using previously published semiquantitative scale (Fig. 1) [11,12]. FLAIR image was used for the assessment and the hyperintensity was classified into two categories, those in periventricular white matter and those in deep white matter. When a periventricular cap or band was found, those lesions were defined as periventricular white matter hyperintensity (PWMH). The size of the cap was measured along the longest length from the anterior or posterior horn on a perpendicular axis. The size of the band was measured along the longest length from the lateral wall of the ventricle on the horizontal axis. Both cap and band were rated as P1 ( $<5 \mathrm{~mm}$ ), P2 (5 to $9 \mathrm{~mm}$ ), or P3 ( $\geq 10 \mathrm{~mm}$ ). Deep white matter hyperintensity (DWMH) was defined as white matter hyperintensity lesion found above the second cut from the upper margin of the ventricle on FLAIR images, or close to the lateral ventricle with normal white matter between them. DWMH was rated as D1 (small and large foci, $<10 \mathrm{~mm}$ ), D2 (beginning of a confluent lesion,
10 to $24 \mathrm{~mm}$ ), or D3 (confluent lesion, $\geq 25 \mathrm{~mm}$ ). Infarction lesions, both acute and chronic state, were excluded from the assessment. Lacunar infarction was also excluded, defined as following; size 3 to $15 \mathrm{~mm}$, T2 high signal, FLAIR low signal with gliotic rim, and T1 low signal [9].

\section{Statistical analysis}

The differences of clinical characteristics between subgroups were tested with one way analysis of variance (ANOVA) for continuous variables and chi-square test for categorical variables. To investigate associations between the clinical factors and cerebral white matter hyperintensity, both univariable and multivariable ordinal regression analysis were performed. In multivariable ordinal regression analysis model, variables with $\mathrm{P}<0.10$ in univariable analysis and clinically important factors such as BMD, alkaline phosphatase (ALP), phosphorus, calcium, and glomerular filtration rate (GFR) were included and backward elimination method was used. All statistical analyses were performed using SPSS version 20.0 (IBM Co., Ar-

Table 1. Clinical characteristics of subject subgroups according to PWMH and DWMH grade

\begin{tabular}{|c|c|c|c|c|c|c|c|c|}
\hline \multirow{2}{*}{ Characteristic } & \multicolumn{4}{|c|}{ PWMH } & \multicolumn{4}{|c|}{ DWMH } \\
\hline & Grade 1 & Grade 2 & Grade 3 & P-value & Grade 1 & Grade 2 & Grade 3 & P-value \\
\hline Age (yr) & $73.48 \pm 5.40$ & $75.21 \pm 5.90$ & $77.39 \pm 6.59$ & $<0.001^{\mathrm{a})}$ & $74.07 \pm 5.44$ & $75.75 \pm 6.24$ & $78.55 \pm 6.88$ & $<0.001^{\text {a) }}$ \\
\hline Male sex & $74(59.7)$ & $81(52.3)$ & $58(45.0)$ & 0.064 & $123(60.6)$ & $66(47.8)$ & $24(35.8)$ & $0.001^{\text {a) }}$ \\
\hline Weight (kg) & $62.42 \pm 9.90$ & $60.50 \pm 9.95$ & $59.23 \pm 11.02$ & 0.053 & $61.69 \pm 10.04$ & $59.52 \pm 10.10$ & $60.13 \pm 11.51$ & 0.154 \\
\hline Height $(\mathrm{cm})$ & $162.07 \pm 8.30$ & $160.32 \pm 8.94$ & $159.27 \pm 8.84$ & $0.038^{\mathrm{a})}$ & $161.90 \pm 8.38$ & $159.38 \pm 9.00$ & $158.61 \pm 8.88$ & $0.005^{\mathrm{a})}$ \\
\hline $\mathrm{BMI}\left(\mathrm{kg} / \mathrm{m}^{2}\right)$ & $23.63 \pm 2.89$ & $23.37 \pm 2.95$ & $23.26 \pm 2.99$ & 0.610 & $23.40 \pm 2.95$ & $23.20 \pm 2.72$ & $23.93 \pm 3.33$ & 0.277 \\
\hline BMD (T-score) & $-2.06 \pm 1.16$ & $-2.43 \pm 1.12$ & $-2.82 \pm 1.05$ & $<0.001^{\mathrm{a})}$ & $-2.17 \pm 1.16$ & $-2.65 \pm 1.09$ & $-2.82 \pm 1.06$ & $<0.001^{\text {a) }}$ \\
\hline \multicolumn{9}{|l|}{ Clinical history } \\
\hline Hypertension & $84(68.3)$ & $126(81.3)$ & $103(79.8)$ & $0.024^{\mathrm{a})}$ & $148(72.9)$ & 109 (79.6) & $56(83.6)$ & 0.132 \\
\hline Diabetes mellitus & $44(35.8)$ & $63(40.6)$ & $42(32.8)$ & 0.384 & 80 (39.4) & $49(36.0)$ & $20(29.9)$ & 0.364 \\
\hline Atrial fibrillation & $40(32.3)$ & $31(20.0)$ & $26(20.2)$ & $0.029^{\mathrm{a})}$ & $57(28.1)$ & 27 (19.6) & $13(19.4)$ & 0.127 \\
\hline Smoking & $31(25.2)$ & $37(23.9)$ & $29(22.7)$ & 0.891 & $54(26.6)$ & $32(23.5)$ & $11(16.4)$ & 0.259 \\
\hline Previous stroke & $14(11.3)$ & $13(8.4)$ & $18(14.0)$ & 0.327 & $19(9.4)$ & $15(10.9)$ & $11(16.4)$ & \\
\hline \multicolumn{9}{|l|}{ Laboratory test } \\
\hline $\operatorname{ALP}(I U / L)$ & $77.89 \pm 27.37$ & $72.49 \pm 20.07$ & $80.94 \pm 27.47$ & $0.016^{\mathrm{a})}$ & $76.25 \pm 25.82$ & $76.16 \pm 26.56$ & $79.72 \pm 19.10$ & 0.580 \\
\hline Phosphorus (mg/dL) & $3.13 \pm 0.63$ & $3.21 \pm 0.48$ & $3.16 \pm 0.51$ & 0.402 & $3.17 \pm 0.58$ & $3.18 \pm 0.51$ & $3.15 \pm 0.48$ & 0.914 \\
\hline Total calcium (mg/dL) & $8.85 \pm 0.37$ & $8.95 \pm 0.46$ & $8.86 \pm 0.44$ & 0.076 & $8.93 \pm 0.41$ & $8.87 \pm 0.46$ & $8.81 \pm 0.44$ & 0.116 \\
\hline $\mathrm{GFR}^{\mathrm{b})}(\mathrm{mL} / \mathrm{min})$ & $89.80 \pm 16.41$ & $83.01 \pm 16.69$ & $81.13 \pm 16.88$ & $<0.001^{\text {a) }}$ & $86.12 \pm 17.32$ & $83.46 \pm 17.23$ & $81.57 \pm 15.20$ & 0.114 \\
\hline $\mathrm{LDL}-\mathrm{C}(\mathrm{mg} / \mathrm{dL})$ & $105.38 \pm 28.22$ & $105.80 \pm 32.47$ & $108.56 \pm 29.69$ & 0.661 & $104.90 \pm 29.34$ & $106.23 \pm 31.56$ & $112.11 \pm 30.42$ & 0.245 \\
\hline $\mathrm{HDL}-\mathrm{C}(\mathrm{mg} / \mathrm{dL})$ & $45.24 \pm 10.32$ & $44.83 \pm 11.10$ & $45.30 \pm 11.95$ & 0.926 & $45.37 \pm 10.61$ & $44.31 \pm 12.31$ & $45.92 \pm 10.10$ & 0.557 \\
\hline Triglyceride (mg/dL) & $98.88 \pm 39.57$ & $117.46 \pm 55.68$ & $105.38 \pm 49.28$ & $0.007^{\mathrm{a})}$ & $106.15 \pm 48.65$ & $113.33 \pm 52.13$ & $102.35 \pm 47.28$ & 0.267 \\
\hline Homocysteine ( $\mu \mathrm{mol} / \mathrm{L})$ & $9.78 \pm 3.27$ & $11.12 \pm 4.09$ & $12.39 \pm 4.61$ & $<0.001^{\mathrm{a})}$ & $10.85 \pm 4.06$ & $11.48 \pm 4.24$ & $11.16 \pm 4.25$ & 0.397 \\
\hline Lipoprotein- $\alpha$ (mg/dL) & $3.62 \pm 1.54$ & $3.74 \pm 1.38$ & $3.68 \pm 1.46$ & 0.803 & $3.63 \pm 1.52$ & $3.75 \pm 1.48$ & $3.70 \pm 1.45$ & 0.756 \\
\hline
\end{tabular}

Values are presented as mean \pm standard deviation or number (\%). Analysis of variance (ANOVA) for continuous variables and chi-square test for categorical variables were performed.

PWMH, periventricular white matter hyperintensity; DWMH, deep white matter hyperintensity; BMI, body mass index; BMD, bone mineral density; ALP, alkaline phosphatase; GFR, glomerular filtration rate; LDL-C, low density lipoprotein cholesterol; HDL-C, high density lipoprotein cholesterol.

${ }^{a)} \mathrm{P}<0.05$; ${ }^{\text {b) }}$ Calculated with creatinine, age, sex, and weight. 
monk, NY, USA), R language version 3.01 (R Foundation for Statistical Computing, Vienna, Austria), or SAS version 9.1 (SAS Institute Inc., Cary, NC, USA), and P-values $<0.05$ were regarded as significant.

\section{RESULTS}

Finally, 408 patients were included in the study; $75.37 \pm 6.17$ years old and 213 (52.2\%) were male. Mean BMD T-score was $-2.439 \pm 1.15$ and 202 (49.5\%) had osteoporosis, T-score lower than -2.5. Distribution of PWMH subgroup was as follows; 124 for grade 1, 155 for grade 2, and 129 for grade 3. Distribution of DWMH subgroup was as follows; 203 for grade 1, 138 for grade 2, and 67 for grade 3 . In comparison of clinical characteristics among PWMH subgroups, age, height, BMD, hypertension history, atrial fibrillation (AF) history, triglyceride, homocysteine, ALP, and GFR were significantly different. In comparison of clinical characteristics among DWMH subgroups, age, sex, height, and BMD were significantly different (Table 1).

In univariable regression analysis of PWMH as a response variable, age, sex, weight, height, BMD, hypertension history, AF history, GFR, and homocysteine showed significant association with P-value less than 0.1 (Supplementary Table 1). In univariable regression analysis of DWMH as a response variable, age, sex, weight, height, BMD, hypertension history, AF history, total calcium, and GFR showed P-value less than 0.1 (Table 1). In multivariable regression analysis, BMD was signifi- cantly correlated with both PVMH (odd ratio [OR], -0.416; 95\% confidence interval $[\mathrm{Cl}],-0.654$ to $-0.178 ; \mathrm{P}=0.001)$ and $\mathrm{DVMH}$ (OR, 0.306; $95 \% \mathrm{Cl},-0.546$ to $-0.067 ; \mathrm{P}=0.012$ ) (Table 2). Changes of PWMH and DWMH grades exert synergistic effect on BMD (Fig. 2). In addition, several factors showed significant correlations; old age and high serum homocysteine for high PWMH grade; old age, AF history, and high serum total calcium for high DWMH grade.

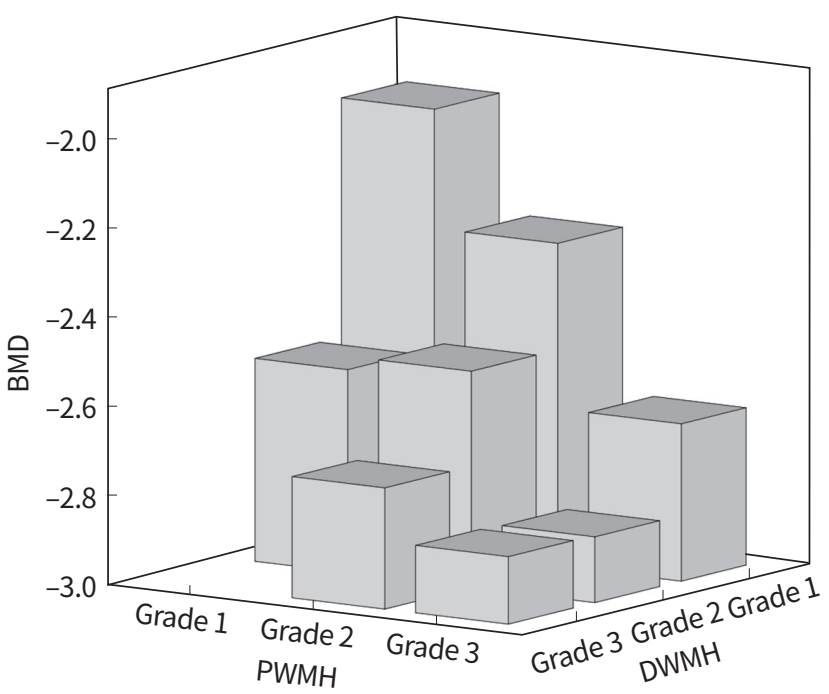

Fig. 2. Mean bone mineral density (BMD) of subgroups, classified with the grade of periventricular white matter hyperintensity (PWMH) and deep white matter hyperintensity (DWMH). According to increase of grade, BMD tends to decrease. There was no subjects with $\mathrm{PWMH}$ grade 1 and $\mathrm{DWMH}$ grade 3 .

Table 2. Relationships between clinical factors and subtypes of cerebral white matter hyperintensity

\begin{tabular}{|c|c|c|c|c|c|c|}
\hline \multirow{2}{*}{ Variable } & \multicolumn{3}{|c|}{ PWMH } & \multicolumn{3}{|c|}{ DWMH } \\
\hline & OR & $95 \% \mathrm{Cl}$ & P-value & OR & $95 \% \mathrm{Cl}$ & P-value \\
\hline Age $^{a, b)}$ & 0.037 & 0.002 to 0.073 & $0.040^{c)}$ & 0.057 & 0.021 to 0.092 & $0.002^{c)}$ \\
\hline $\operatorname{Sex}^{\mathrm{a}(\mathrm{b}) \mathrm{b}}$ & 0.222 & -0.387 to 0.831 & 0.475 & 0.401 & -0.108 to 0.910 & 0.122 \\
\hline Weight $^{\text {b) }}$ & - & - & - & 0.018 & -0.006 to 0.041 & 0.141 \\
\hline Height $^{\text {a) }}$ & 0.011 & -0.022 to 0.045 & 0.508 & - & - & - \\
\hline $\mathrm{BMD}^{\mathrm{a}, \mathrm{b})}$ & -0.427 & -0.654 to -0.199 & $<0.001^{\text {c) }}$ & -0.326 & -0.563 to -0.088 & $0.007^{c)}$ \\
\hline Hypertensiona),b) & 0.281 & -0.199 to 0.761 & 0.251 & 0.257 & -0.243 to 0.756 & 0.314 \\
\hline Atrial fibrillation a),b) & -0.436 & -0.909 to 0.037 & 0.071 & -0.634 & -1.124 to -0.145 & $0.011^{c)}$ \\
\hline$G_{F R}{ }^{a, b)}$ & -0.004 & -0.020 to 0.011 & 0.592 & -0.009 & -0.022 to 0.004 & 0.188 \\
\hline Homocysteine $e^{\text {a) }}$ & 0.123 & 0.063 to 0.182 & $<0.001^{\mathrm{c})}$ & - & - & - \\
\hline ALP(,), & 0.001 & -0.008 to 0.010 & 0.820 & 0.002 & -0.006 to 0.010 & 0.695 \\
\hline Phosphorus & - & - & - & -0.147 & -0.543 to 0.248 & 0.465 \\
\hline Total calcium a),b) & -0.146 & -0.022 to 0.045 & 0.508 & -0.474 & -0.945 to -0.002 & 0.049 \\
\hline
\end{tabular}

Multivariable ordinal regression analysis was performed.

$\mathrm{PWMH}$, periventricular white matter hyperintensity; DWMH, deep white matter hyperintensity; OR, odd ratio; $\mathrm{Cl}$, confidence interval; $\mathrm{BMD}$, bone mineral density; GFR, glomerular filtration rate; ALP, alkaline phosphatase.

a) Variables for $\mathrm{PWMH} ;{ }^{\mathrm{b})}$ Variables for $\mathrm{DWMH} ;{ }^{\mathrm{C})} \mathrm{P}<0.05$ was presented as bold characters. 


\section{DISCUSSION}

In the present study, severity of cerebral white matter hyperintensity on MRI showed significant associations with BMD, compatible results with the previous study. Previous study showed significant association between presence of osteoporosis and cerebral SVD on CT [7]. Although other previous study stated no significant correlation between SVD and low BMD, the study used phalynx rather than femur neck as measuring site of BMD [13]. Because phalynx is not a recommended site for BMD measuring to assess osteoporosis in clinical practice, BMD measured in phalynx maybe insufficiently reflect the presence of osteoporosis [14].

It is suggested that sharing of risk factor might explain the association between osteoporosis and cerebral SVD [7]. Traditionally, cerebral SVD that refers to atherosclerosis has been known as having risk factors such as aging, hypertension, diabetes mellitus, dyslipidemia, smoking, or homocysteine [15]. Osteoporosis has several risk factors similar to cerebral SVD in part, like smoking and old age. However, in this study, the association between cerebral white matter hyperintensity and BMD was independent of such risk factors, thus the association could not be explained by risk factors sharing. Although the underlying pathophysiologic mechanisms between osteoporosis and cerebral SVD are still on debate, one of the possible explanations is that osteoporosis-related abnormal calcium metabolism could increase vascular calcification and arterial wall stiffness [5,16]. Vascular calcification has been suggested as a marker of cerebral SVD in recent observations $[17,18]$. The association may also be explained by common pathophysiology of the two conditions. Atherosclerosis, a main pathology of cerebral SVD, and osteoporosis are known to have common pathogenesis like bone morphogenetic proteins, the receptor activator of nuclear factor-kappaB ligand (RANKL)/RANK/osteoprotegerin (OPG) pathway, matrix Gla protein (MGP) and vitamin K [19]. Low vitamin $D$ level also is known to be associated with increased vascular risk [20].

If osteoporosis is an independent risk factor of cerebral SVD, we can assume that treatment of osteoporosis reduces the occurrence or progression of cerebral SVD. However, such concept should be considered with caution. Two well-established agents for treating osteoporosis, bisphosphonate, and calcium, showed a dissimilar influence to atherosclerosis. There has been no consensus about the effect of bisphosphonate on atherosclerosis. Several observational studies demonstrated that bisphosphonate treatment had benefit on reducing the risk of cardiovascular disease such as myocardial infarction or stroke $[21,22]$. Bisphosphonate has been suggested to reduce cardiovascular disease by the following mechanisms; inhibition of intimal hyperplasia, macrophage-related inflammation, and vascular calcification [22,23]. However, recent meta-analysis showed different results that bisphosphonate do not act as beneficial or harmful factor on atherosclerosis [24]. Meanwhile, calcium supplements may be harmful to cardiovascular disease $[25,26]$. Contrast to daily calcium intake dose, artificial calcium supplements lead to significant increase of serum calcium level. The increased calcium level is associated with increased cardiovascular risk $[25,27,28]$. Whether active treatment of osteoporosis reduces the risk of cerebral SVD is not clear and careful approach is needed.

In addition to BMD, several factors were associated with cerebral SVD. Age was independent risk factor for both PWMH and DWMH, as reported in previous study [29]. AF was inversely correlated with DWMH $(P=0.011 ; O R,-0.634)$. Although statistically not significant, $\mathrm{PWMH}$ also showed tendency of negative association with $A F(P=0.071 ; O R,-0.436)$. The results can be explained by the following. The study was performed with acute symptomatic stroke patients. AF is a major cause of cardioembolic stroke, and patients with cardioembolic stroke have smaller cerebral white matter hyperintensity volume than those with large artery atherosclerosis or small vessel occlusion $[30,31]$. Homocysteine showed significant association with PVMH but not with DVMH. In the contrast, serum calcium level is associated with DVMH only. Discrepancy of significant association factors between PWMH and DWMH may indicate that the two categories have different pathomechanism in part, which is not clearly identified, and reserved for future investigation.

The present study has several limitations. First, the study population consisted of a single-race, was selected from hospitalized symptomatic stroke patients and healthy subjects were not included. Thus there are some limitations to generalize the results. Second, subjects taking osteoporosis medications were excluded for the study. The strict inclusion criteria was unavoidable due to more homogeneous study populations; however, subjects with severe or symptomatic osteoporosis may be eliminated from the study and it can attenuate the results. Finally, MRI equipment used for the study was not identical to each other, one was 1.5 Tesla and the other was 3.0 Tesla. Because measurement of cerebral white matter intensities is a precision task and requires high resolution images, heterogeneity of MRI equipment may influence to measured size of cerebral white matter intensities. However, possible missing or mis-sized lesions on low tesla MRI are of- 
ten small-sized; hence, they do not significantly influence to grading of cerebral white matter hyperintensity, because the grade in the study was decided by size of biggest lesion [32].

In conclusion, low BMD was associated with a high severity of cerebral white matter hyperintensity in elderly Asian stroke patients. This association was independent of a shared etiology of osteoporosis and cerebral SVD. Osteoporosis could be linked to dementia or stroke through cerebral SVD, it would be important to screen osteoporosis in advance. But the effect of osteoporosis treatment on cerebral SVD is still on debate and further longitudinal studies are requested.

\section{CONFLICTS OF INTEREST}

No potential conflict of interest relevant to this article was reported.

\section{REFERENCES}

1. Prevention and management of osteoporosis. World Health Organ Tech Rep Ser 2003;921:1-164.

2. Minn YK, Suk SH, Do SY. Osteoporosis as an independent risk factor for silent brain infarction and white matter changes in men and women: the PRESENT project. Osteoporos Int 2014;25:2465-9.

3. Lampropoulos CE, Papaioannou I, D'Cruz DP. Osteoporosis: a risk factor for cardiovascular disease? Nat Rev Rheumatol 2012;8:587-98.

4. Bolland MJ, Avenell A, Baron JA, Grey A, MacLennan GS, Gamble GD, et al. Effect of calcium supplements on risk of myocardial infarction and cardiovascular events: meta-analysis. BMJ 2010;341:c3691.

5. Santos LL, Cavalcanti TB, Bandeira FA. Vascular effects of bisphosphonates-a systematic review. Clin Med Insights Endocrinol Diabetes 2012;5:47-54.

6. Kang JH, Keller JJ, Lin HC. Bisphosphonates reduced the risk of acute myocardial infarction: a 2-year follow-up study. Osteoporos Int 2013;24:271-7.

7. Carda S, Cisari C, Invernizzi M, Bevilacqua M. Osteoporosis after stroke: a review of the causes and potential treatments. Cerebrovasc Dis 2009;28:191-200.

8. Meyer JS, Kawamura J, Terayama Y. White matter lesions in the elderly. J Neurol Sci 1992;110:1-7.

9. Wardlaw JM, Brindle W, Casado AM, Shuler K, Henderson M, Thomas B, et al. A systematic review of the utility of 1.5 versus 3 Tesla magnetic resonance brain imaging in clinical practice and research. Eur Radiol 2012;22:2295-303.
10. Alagiakrishnan K, Hsueh J, Zhang E, Khan K, Senthilselvan $A$. Small vessel disease/white matter disease of the brain and its association with osteoporosis. J Clin Med Res 2015;7:297-302.

11. Kim DH, Rogers JR, Fulchino LA, Kim CA, Solomon DH, Kim SC. Bisphosphonates and risk of cardiovascular events: a meta-analysis. PLoS One 2015;10:e0122646.

12. Foley RN, Collins AJ, Ishani A, Kalra PA. Calcium-phosphate levels and cardiovascular disease in community-dwelling adults: the Atherosclerosis Risk in Communities (ARIC) Study. Am Heart J 2008;156:556-63.

13. Kang JH, Keller JJ, Lin HC. A population-based 2-year follow-up study on the relationship between bisphosphonates and the risk of stroke. Osteoporos Int 2012;23:25517.

14. Noh Y, Lee Y, Seo SW, Jeong JH, Choi SH, Back JH, et al. A new classification system for ischemia using a combination of deep and periventricular white matter hyperintensities. J Stroke Cerebrovasc Dis 2014;23:636-42.

15. Rost NS, Rahman RM, Biffi A, Smith EE, Kanakis A, Fitzpatrick $\mathrm{K}$, et al. White matter hyperintensity volume is increased in small vessel stroke subtypes. Neurology 2010; 75:1670-7.

16. Choi JY, Seo WK, Kang SH, Jung JM, Cho KH, Yu S, et al. Statins improve survival in patients with cardioembolic stroke. Stroke 2014;45:1849-52.

17. Seo WK, Lee JM, Park MH, Park KW, Lee DH. Cerebral microbleeds are independently associated with arterial stiffness in stroke patients. Cerebrovasc Dis 2008;26:61823.

18. Mussolino ME, Madans JH, Gillum RF. Bone mineral density and stroke. Stroke 2003;34:e20-2.

19. Hong NR, Seo HS, Lee YH, Kim JH, Seol HY, Lee NJ, et al. The correlation between carotid siphon calcification and lacunar infarction. Neuroradiology 2011;53:643-9.

20. Poole KE, Reeve J, Warburton EA. Falls, fractures, and osteoporosis after stroke: time to think about protection? Stroke 2002;33:1432-6.

21. Wardlaw JM, Smith C, Dichgans M. Mechanisms of sporadic cerebral small vessel disease: insights from neuroimaging. Lancet Neurol 2013;12:483-97.

22. Frost ML, Grella R, Millasseau SC, Jiang BY, Hampson G, Fogelman I, et al. Relationship of calcification of atherosclerotic plaque and arterial stiffness to bone mineral density and osteoprotegerin in postmenopausal women referred for osteoporosis screening. Calcif Tissue Int 2008;83:112 20. 
23. Jorde R, Sundsfjord J, Fitzgerald P, Bonaa KH. Serum calcium and cardiovascular risk factors and diseases: the Tromso study. Hypertension 1999;34:484-90.

24. lijima K, Akishita M, Ouchi Y. Coronary artery calcification and cerebral small vessel disease. Association of systemic atherosclerosis. Circ J 2011;75:272-3.

25. Lee SB, Cho AH, Butcher KS, Kim TW, Ryu SY, Kim YI. Low bone mineral density is associated with poor clinical outcome in acute ischemic stroke. Int J Stroke 2013;8:68-72.

26. Myint PK, Clark AB, Kwok CS, Loke YK, Yeong JK, Luben RN, et al. Bone mineral density and incidence of stroke: European prospective investigation into cancer-norfolk population-based study, systematic review, and meta-analysis. Stroke 2014;45:373-82.

27. Wardlaw JM, Smith EE, Biessels GJ, Cordonnier C, Fazekas $F$, Frayne R, et al. Neuroimaging standards for research into small vessel disease and its contribution to ageing and neurodegeneration. Lancet Neurol 2013;12:822-38.

28. Golob AL, Laya MB. Osteoporosis: screening, prevention, and management. Med Clin North Am 2015;99:587-606.

29. Beveridge LA, Witham MD. Vitamin D and the cardiovascular system. Osteoporos Int 2013;24:2167-80.

30. Wahlund LO, Barkhof F, Fazekas F, Bronge L, Augustin M, Sjogren $M$, et al. A new rating scale for age-related white matter changes applicable to MRI and CT. Stroke 2001;32: 1318-22.

31. Bolland MJ, Barber PA, Doughty RN, Mason B, Horne A, Ames R, et al. Vascular events in healthy older women receiving calcium supplementation: randomised controlled trial. BMJ 2008;336:262-6.

32. Chutinet A, Biffi A, Kanakis A, Fitzpatrick KM, Furie KL, Rost NS. Severity of leukoaraiosis in large vessel atherosclerotic disease. AJNR Am J Neuroradiol 2012;33:1591-5. 
Supplementary Table 1. Univariable regression analysis between clinical factors and cerebral white matter hyperintensity subtypes

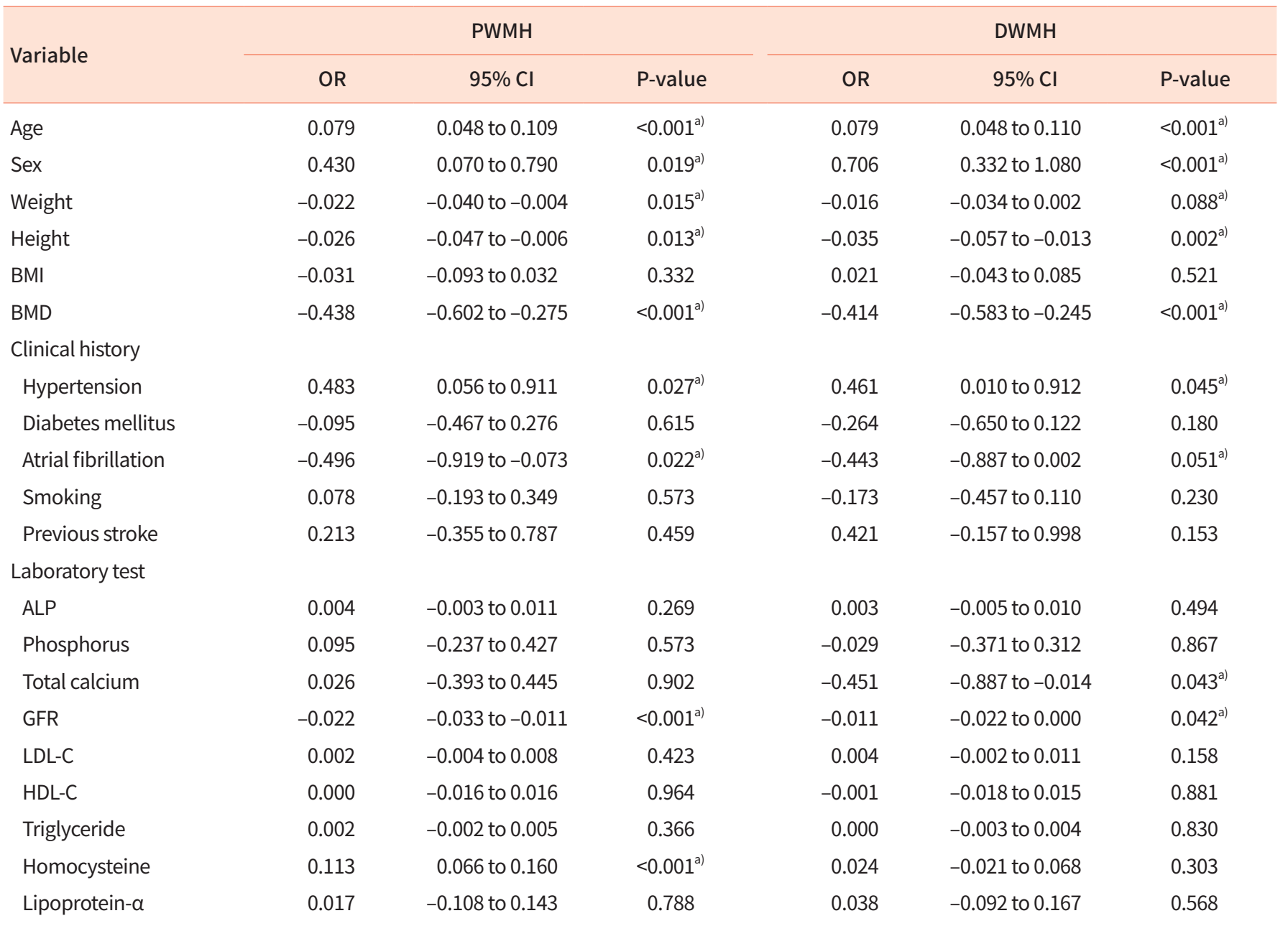

Univariable ordinal regression analysis was performed.

PWMH, periventricular white matter hyperintensity; DWMH, deep white matter hyperintensity; OR, odd ratio; $\mathrm{Cl}$, confidence interval; BMI, body mass index; BMD, bone mineral density; ALP, alkaline phosphatase; GFR, glomerular filtration rate; LDL-C, low density lipoprotein cholesterol; HDL-C, high density lipoprotein cholesterol.

${ }^{\text {a) }} \mathrm{P}<0.1$. 